\title{
Research on incentive mechanism of benefit distribution in collaborative innovation of industry alliance
}

\author{
Yue $\mathrm{Li}^{1, *}$, Jiepeng Huang ${ }^{1}$, Hang Guo ${ }^{1}$ and Zhuo Wang ${ }^{1}$ \\ ${ }^{1}$ School of Economics and Management, Harbin University of Science and Technology, 150080 \\ Harbin, Heilongjiang, China
}

\begin{abstract}
In order to improve the performance of alliance collaborative innovation and stimulate members' willingness and behavior to participate in collaborative innovation, this paper puts forward the incentive mechanism of benefit distribution. This paper divides the needs of members to participate in collaborative innovation into two stages: "risk avoidancereturn on investment". Firstly, an effective benefit distribution model is established by using Logistic function. Then, by building a game model, we can get the best effort. The results show that building the benefit distribution model of alliance collaborative innovation according to the different needs of alliance members can fully stimulate members to participate in collaborative innovation and improve the performance of alliance collaborative innovation.
\end{abstract}

\section{Introduction}

Collaborative innovation is an important means for alliance members to realize key technological innovations. Because technological innovation activities are faced with greater resource input and higher risk, individual enterprises cannot achieve their goals in a short time by relying on their own resources, and alliance collaborative innovation provides an effective way. However, in the process of alliance collaborative innovation, it still shows a lot of instability, which can not achieve the expected performance of alliance collaborative innovation, resulting in the forced dissolution of some alliances. The main reasons are: on the one hand, the satisfaction with the results of benefit distribution is not high, which leads to the low performance of alliance collaborative innovation; On the other hand, different alliance members have different interests and needs to participate in collaborative innovation, which leads to the inability to work together for the same goal. Therefore, the key to the incentive mechanism of benefit distribution in alliance collaborative innovation lies in taking the benefit demand of alliance members as the basis, and then improving the satisfaction of alliance members to the benefit distribution results.

At present, the research on incentive mechanism of benefit distribution mainly focuses on the construction of distribution model and optimization of distribution results. Reasonable and perfect distribution of benefits is the return of efforts and resources of alliance members,

* Corresponding author: lykusha@,163.com 
and is the premise and guarantee of efficient operation of alliance. In the process of output distribution, members' input and output distribution, the demand and preference for resources and the maximization of members' overall utility are the main considerations (Wang Xueyuan, $2011)^{[1]}$. Zhou Qing et al (2015) put forward four ways: distribution according to investment proportion, distribution according to actual contribution in cooperation process, distribution according to paid purchase technology and distribution according to free use of technology by enterprises ${ }^{[2]}$. Wang Xiang et al (2014) combined Shapley cooperative game (Li Gang, 2010) and Pareto optimal non-cooperative game (Bo Huang et al, 2010) to design a balanced distribution mode between enterprises within the alliance and between enterprises and external supervisors through third-party supervision institutions ${ }^{[3-7]}$.

Through combing the literature, it is found that many studies have been carried out on the construction of distribution model and optimization of results at home and abroad, but most of them focus on distribution methods and models, and there is a lack of in-depth research on the process of distribution of benefits based on the needs of alliance members. Based on the interests of alliance members, this paper establishes an effective incentive mechanism for collaborative innovation through Logic function and game model method. It provides a scientific basis for the alliance to further enhance the incentive effect of benefit distribution, and provides a reference theory and method for further improving the collaborative innovation performance of the alliance.

\section{Benefit function of alliance collaborative innovation}

\subsection{Basic assumptions}

According to the process and law of participating in collaborative innovation and cooperation among the members of the Alliance, the following basic assumptions are put forward:

(1) $\mathrm{N}=\{1,2, \cdots \mathrm{n}\}$ is set up as a collection of members of the alliance's collaborative innovation cooperation, in which interest subject $i(i=1,2, \cdots n)$ expects to bring innovation benefits to itself through collaborative innovation and cooperation, and the demand for benefit distribution results is obviously different at different stages of collaborative innovation..

(2) The benefits of collaborative innovation of alliance are mainly realized through the efforts and interactions of members. The efforts are mainly reflected in the input of funds, personnel, equipment, knowledge, technology and other resources in the process of collaborative innovation. Interaction intensity is mainly reflected in the frequency of information exchange and the degree of coordination of behavior among alliance members. Government policies, laws and regulations, and market environment, which have an impact on the alliance's collaborative innovation performance, are uncertain and subject to a normal distribution, i.e. $x \sim N\left(0, \sigma^{2}\right)$.

(3) The effort and interaction intensity of alliance members can be comprehensively measured by their participation in collaborative innovation and their contribution to collaborative innovation results, which can be used as an important indicator for the alliance to motivate its members through the benefit distribution process.

(4) Alliance collaborative innovation is a long-term cooperation mode established among members, and their risk preferences are different, so it is necessary to distribute their ownership after realizing the phased achievements of alliance collaborative innovation. With the smooth progress of cooperation, it is necessary to design a reasonable benefit distribution mode for all perfect innovation achievements by means of transforming value. 


\subsection{Benefit function}

According to the traditional benefit accounting method, the benefit of alliance collaborative innovation is the difference between the performance and cost of alliance collaborative innovation, in which the innovation cost is calculated by self-innovation cost and alliance cooperative innovation cost respectively. ${ }^{[8,9]}$

Definition 1 Set $N=\{1,2, \cdots, n\}$ as a collection of alliance collaborative innovation members, $i(i=1,2, \cdots, n)$ 's efforts are $T i$ and the intensity of interaction of member is $W_{i}$.

In the initial stage of collaborative innovation cooperation, the efforts of alliance members are mainly reflected by the input of resources such as capital, personnel, equipment, knowledge and technology. However, the efforts of members themselves can not achieve the collaborative innovation performance of alliance alone, and the interaction between members is needed to transform the resource input into performance, which are the key factors affecting the performance level of collaborative innovation.

Definition 2 The performance, cost, and benefit of the alliance members' collaborative innovation are all functions of the member's effort level $T_{i}$, recorded as $S_{i}, C_{i}$, and $V_{i}$. According to the basic connotation of Douglas production function, the function relationship between member i 's collaborative innovation performance $\mathrm{Si}$ and effort level $T_{i}$ and interaction intensity $W_{i}$ is:

$$
\mathrm{S}_{\mathrm{i}}\left(\mathrm{t}_{\mathrm{i}}, \mathrm{w}_{\mathrm{i}}\right)=\alpha \mathrm{t}_{\mathrm{i}}^{\mathrm{a}} \mathrm{w}_{\mathrm{i}}^{\mathrm{b}}(0<\mathrm{a}<1,0<\mathrm{b}<1, \mathrm{i}=1,2, \cdots, \mathrm{n})
$$

Among them, parameter $\beta_{i}$ is the yield elasticity coefficient of member $i$ effort, and parameter $b$ is the yield elasticity coefficient of member $i$ interaction intensity. $\alpha$ is the trust parameter between the members of the alliance, which is compared to the comprehensive technical level coefficient in the Cobb Douglas function.

Definition 3 Drawing lessons from Zhang Weiying's quantitative research process on effort cost, $\beta_{i}$ is the effort cost coefficient in the collaborative innovation process of members, and the cost consumed by member $i$ at effort level $T_{i}$ is:

$$
\mathrm{C}_{\mathrm{i}}\left(\mathrm{t}_{\mathrm{i}}\right)=\frac{1}{2} \beta_{\mathrm{i}} \mathrm{t}_{\mathrm{i}}^{2},(\mathrm{i}=1,2, \cdots, \mathrm{n})
$$

The cost of collaborative innovation of alliance members mainly comes from the efforts of members in the process of cooperation, that is, the cost caused by the input of resources. The increased efforts of alliance members indicate that the amount of resources invested increases, resulting in an increase in innovation costs.

Theorem 1 Under the effect of effort level $T_{i}$ and interaction intensity $W_{i}$ of alliance member $i$, the expected return function of collaborative innovation is:

$$
\mathrm{EV}_{\mathrm{i}}\left(\mathrm{t}_{\mathrm{i}}, \mathrm{w}_{\mathrm{i}}\right)=\alpha \mathrm{t}_{\mathrm{i}}^{\mathrm{a}} \mathrm{w}_{\mathrm{i}}^{\mathrm{b}}-\frac{1}{2} \beta_{\mathrm{i}} \mathrm{t}_{\mathrm{i}}{ }^{2},(\mathrm{i}=1,2, \cdots, \mathrm{n})
$$

Definition 4 The overall performance, costs and benefits of alliance collaborative innovation are represented by S, C, and V respectively. In the overall performance of the alliance's collaborative innovation, taking into account the gradual deepening of cooperation between members, the degree of trust within the alliance $\alpha$, the effort gain coefficient a, and the interaction gain coefficient b will change. That is, $\alpha \rightarrow \alpha^{\prime}, a \rightarrow a^{\prime}, b \rightarrow b^{\prime}$, where $\alpha<$ $\alpha^{\prime}, a<a^{\prime}, b<b^{\prime}$. The overall performance function of alliance collaborative innovation is:

$$
\mathrm{S}\left(\mathrm{t}_{\mathrm{i}}, \mathrm{w}_{\mathrm{i}}\right)=\sum_{\mathrm{i}=\mathrm{i}}^{\mathrm{n}}\left(\alpha^{\prime} \mathrm{t}_{\mathrm{i}}^{\mathrm{a}^{\prime}} \mathrm{w}_{\mathrm{i}}^{\mathrm{b}^{\prime}}\right),(\mathrm{i}=1,2, \cdots, \mathrm{n})
$$


Definition 5 In the process of collaborative innovation, alliance members complement each other and share the cost, which makes the effort cost coefficient of alliance members to be $\beta_{i}>\beta_{i}{ }^{\prime}$. Therefore, the cost of collaborative innovation efforts is:

$$
\mathrm{C}\left(\mathrm{t}_{\mathrm{i}}\right)=\sum_{\mathrm{i}=1}^{\mathrm{n}}\left(\frac{1}{2} \beta_{\mathrm{i}}^{\prime} \mathrm{t}_{\mathrm{i}}^{2}\right),(\mathrm{i}=1,2, \cdots, \mathrm{n})
$$

When calculating the overall benefits of alliance collaborative innovation, the cost of alliance collaborative innovation not only considers the effort cost of alliance members, but also considers the risk cost caused by interaction among alliance members in order to realize the incentive effect of benefit distribution on alliance collaborative innovation members. In fact, the interaction between members is a mutual game between cooperation and competition, which maximizes the overall benefits of the alliance in the process of competition and maintains its core competitive advantage in cooperation. Therefore, in order to encourage alliance members to maintain high interaction intensity in the process of collaborative innovation, and to protect their core advantages, it is necessary to compensate the interaction risks borne by members before the benefit distribution, and then formulate a reasonable benefit distribution mode of alliance collaborative innovation.

Definition 6 The risk cost faced by members in the process of interaction is mainly related to the interaction intensity of members called $W_{i}$ in the process of collaborative innovation, so the interactive risk cost of collaborative innovation of alliance is:

$$
C\left(w_{i}\right)=\sum_{i=1}^{n}\left(\gamma_{i} w_{i}^{2}\right),(i=1,2, \cdots, n)
$$

According to the cost accounting method in definition 3, the cost coefficient of Collaborative Innovation Interaction of alliance is set as $\gamma_{i}$. The cost coefficient of each member is different, which is mainly affected by the internal members.

Definition 7 The total cost of alliance collaborative innovation mainly includes two aspects: one is the level of member cooperation, which includes both the cost of resource input called $C\left(t_{i}\right)$ brought by the degree of effort called $T_{i}$, and the risk cost called $C\left(w_{i}\right)$ caused by the intensity of interaction called $W_{i}$; the other is the cost of alliance operation, which is expressed by $Q$.Therefore, the total cost of alliance collaborative innovation is:

$$
\mathrm{C}\left(\mathrm{t}_{\mathrm{i}}, \mathrm{w}_{\mathrm{i}}\right)=\mathrm{C}\left(\mathrm{t}_{\mathrm{i}}\right)+\mathrm{C}\left(\mathrm{w}_{\mathrm{i}}\right)=\sum_{\mathrm{i}=1}^{\mathrm{n}}\left(\frac{1}{2} \beta_{\mathrm{i}} \mathrm{t}_{\mathrm{i}}{ }^{2}+\gamma_{\mathrm{i}} \mathrm{w}_{\mathrm{i}}{ }^{2}\right)+\mathrm{Q},(\mathrm{i}=1,2, \cdots, \mathrm{n})
$$

Theorem 2 Through the joint efforts and interaction of all members of alliance collaborative innovation, the overall benefit of alliance collaborative innovation is:

$\mathrm{V}\left(\mathrm{t}_{\mathrm{i}}, \mathrm{w}_{\mathrm{i}}\right)=\mathrm{S}\left(\mathrm{t}_{\mathrm{i}}, \mathrm{w}_{\mathrm{i}}\right)-\mathrm{C}\left(\mathrm{t}_{\mathrm{i}}, \mathrm{w}_{\mathrm{i}}\right)=\sum_{\mathrm{i}=1}^{\mathrm{n}}\left(\alpha^{\prime} \mathrm{t}_{\mathrm{i}}{ }^{{ }^{\prime}} \mathrm{w}_{\mathrm{i}} \mathrm{b}^{\prime}-\frac{1}{2} \beta_{\mathrm{i}}{ }^{\prime} \mathrm{t}_{\mathrm{i}}{ }^{2}-\gamma_{\mathrm{i}} \mathrm{w}_{\mathrm{i}}{ }^{2}\right)-\mathrm{Q},(\mathrm{i}=1,2, \cdots, \mathrm{n})$

By constructing the benefit distribution function, firstly, it provides an effective benefit distribution means for alliance collaborative innovation, and provides a good institutional guarantee for alliance collaborative innovation cooperation. Secondly, in the benefit function of alliance collaborative innovation, different members bear different shares, Therefore, before the benefit distribution, it is necessary to make up for the risks borne by alliance members, meet the needs of avoiding risks in the process of alliance collaborative innovation, and then stimulate alliance members' willingness to participate in collaborative innovation, thus laying the foundation for the incentive mechanism of subsequent benefit distribution. 


\section{Benefit distribution model of alliance collaborative innovation}

As a key factor for alliance members to participate in collaborative innovation, the degree of effort directly determines the collaborative innovation performance of alliance. Therefore, in the process of benefit distribution of alliance collaborative innovation, it is necessary to realize the incentive effect on the degree of participating efforts. The design of benefit distribution model should be based on the benefit function of alliance collaborative innovation, so as to meet the goal of members' input and return demand, and realize the incentive effect on the efforts of alliance members to participate in collaborative innovation. The details are as follows:

(1) Nash equilibrium model of alliance collaborative innovation under non-cooperative game. Before alliance members participate in collaborative innovation, there is no cooperation agreement among alliance members, so members mainly determine their own efforts by maximizing their own interests, and no member will change their own strategies to destroy the alliance balance and satisfy individual rationality. Therefore, in the Nash equilibrium, the expected interest $E V_{i}$ of the alliance members themselves has the following relationship:

Let the optimal effort level strategy of the member $i(i=1,2, \cdots, n)$ within the alliance be $t_{i}{ }^{*} \in T_{i}$, and the optimal effort level strategy of other members in the non-cooperative game of the alliance is $t_{-i}^{*}=\left\{t_{1}^{*}, \cdots, t_{i-1}^{*}, t_{i+1}^{*}, \cdots, t_{n}^{*}\right\}$, then there is $t_{i} \in T_{i}$, and $t_{i}^{*} \in$ $\arg \max _{t_{i} \in T_{i}} v_{i}\left\{t_{i}, t_{-i}^{*}\right\}$. Based on the above analysis, we can obtain:

$$
\frac{\partial E V_{i}\left(t_{i}, w_{i}\right)}{\partial t_{i}}=a \alpha t_{i}^{a-1} w_{i}^{b}-\beta_{i} t_{i}=0,(i=1,2, \cdots, n)
$$

The optimal effort level of alliance members in the non-cooperative state is $t_{i}{ }^{*}=$ $\left(\frac{a \alpha w_{i}{ }^{b}}{\beta_{i}}\right)^{\frac{1}{2-a}},(i=1,2, \cdots, n)$. Therefore, under Nash equilibrium, the optimal effort level set of alliance members is:

$$
T^{*}=\left(\left(\frac{a \alpha w_{1} b}{\beta_{2}}\right)^{\frac{1}{2-a}},\left(\frac{a \alpha w_{2} b}{\beta_{2}}\right)^{\frac{1}{2-a}}, \cdots,\left(\frac{a \alpha w_{n}{ }^{b}}{\beta_{n}}\right)^{\frac{1}{2-a}}\right)
$$

Therefore, in the process of collaborative innovation among alliance members, the best effort degree of alliance members depends on the interaction degree, trust level, effort benefit coefficient and cost coefficient of alliance members. At this time, the members of the alliance did not establish a perfect cooperative relationship, and each member made efforts to maximize their own interests, thus realizing the Nash equilibrium of the non-cooperative game within the alliance. When alliance collaborative innovation brings more benefits to alliance members, members are willing to participate in cooperation, otherwise they will not participate in cooperation.

Therefore, when the alliance members determine their own optimal cooperation strategy, it is necessary to establish a reasonable benefit distribution model to encourage members to participate in collaborative innovation, which can ensure that members make continuous efforts in the direction of maximizing the benefits of the alliance and avoid damaging the cooperative interests of the alliance due to their own reasons.

(2) Optimization model of alliance benefit distribution under cooperative game. In fact, alliance collaborative innovation cooperation means that alliance members can optimize their cooperation strategies through cooperative games, and will not actively change their strategies. On this basis, a reasonable benefit distribution model is designed to make members 
satisfied with the benefit distribution results, which can encourage alliance members to improve their trust relationship and improve their own efforts.

The aim of alliance members is to maximize the overall interests of the alliance. Under the condition of "collective rationality", alliance members determine their own optimal cooperation strategy through mutual consultation. According to the maximum benefit of the alliance:

$$
\frac{\partial V\left(t_{i}, w_{i}\right)}{\partial t_{i}}=\sum_{i=1}^{n}\left(\alpha^{\prime} a^{\prime} t_{i}^{a^{\prime}-1} w_{i} b^{\prime}-\beta_{i}{ }^{\prime} t_{i}\right)=0
$$

The optimal effort level of the alliance members is $t_{i}=\left(\frac{\alpha^{\prime} a^{\prime} w_{i}^{b^{\prime}}}{\beta_{i}^{\prime}}\right)^{\frac{1}{2-a^{\prime}}},(i=1,2, \cdots, n)$. When the alliance members coordinate and cooperate with each other, the synergy effect is achieved between each other, and the overall benefits of the alliance's collaborative innovation are also maximized. The best effort set of alliance members is:

$$
T^{* *}=\left(\left(\frac{\alpha^{\prime} a^{\prime} w_{1} b^{\prime}}{\beta_{1}^{\prime}}\right)^{\frac{1}{2-a^{\prime}}},\left(\frac{\alpha^{\prime} a^{\prime} w_{2} b^{\prime}}{\beta_{2}^{\prime}}\right)^{\frac{1}{2-a^{\prime}}}, \cdots,\left(\frac{\alpha^{\prime} a^{\prime} w_{n} b^{\prime}}{\beta_{n}^{\prime}}\right)^{\frac{1}{2-a^{\prime}}}\right)
$$

Because $\alpha<\alpha^{\prime}, \quad a<a^{\prime}, \quad b<b^{\prime}$ and $\beta_{i}{ }^{\prime}<\beta_{i}$, so $\left(\frac{\alpha^{\prime} a^{\prime} w_{i} b^{\prime}}{\beta_{i}{ }^{\prime}}\right)^{\frac{1}{2-a^{\prime}}}>\left(\frac{\alpha a w_{i}{ }^{b}}{\beta_{i}}\right)^{\frac{1}{2-a}}$. It can be seen that the alliance collaborative innovation cooperation method provides members with opportunities to obtain higher returns, and at the same time, it also finds a key incentive point for the benefit distribution method. The main basis for the stage is conducive to meeting the needs of members for return on investment in the collaborative innovation process, and can encourage members to continue to improve their own efforts on the basis of the optimal effort of non-cooperative games to obtain higher returns, thereby realizing the distribution of benefits for members to participate the incentive effect of collaborative innovation.

Therefore, in the process of constructing the alliance benefit distribution mechanism, it is always based on the member's effort level $T_{i}$ and the interaction intensity $W_{i}$. Under different incentive stages and incentive goals, the distribution coefficient $\theta_{i}$ is appropriately adjusted to meet the needs of members for benefit distribution. Therefore, let $\theta_{i}=\left\{\theta_{1}, \theta_{2}, \cdots, \theta_{n}\right\}$ be the benefit distribution coefficient of the members of the alliance, $\theta_{i}=K \frac{t_{i}}{\sum_{i=1}^{n} t_{i}}+$ $(1-K) \frac{w_{i}}{\sum_{i=1}^{n} w_{i}},(i=1,2, \cdots, n)$ where $K$ is the proportion of the member's effort $T_{i}$ in the distribution coefficient, and $0<\theta_{i}<1, \sum_{i=1}^{n} \theta_{i}=1$. Then let $\theta_{i}^{+}$and $\theta_{i}^{-}$be the optimal benefit distribution coefficient and the worst benefit distribution coefficient of member $i, Z_{i}$ is the satisfaction degree of member $i$ with the distribution result, $Z_{i}=\frac{\theta_{i}}{\theta_{i}^{+}}$, the alliance members will set the expected standard for the result of allocation according to their own efforts $T_{i}$ and interaction intensity $W_{i}$, then $Z_{i}^{-}=\frac{\theta_{i}^{-}}{\theta_{i}^{+}}$is the minimum standard for alliance members to participate in collaborative innovation, otherwise the members withdraw from the alliance for collaborative innovation cooperation. Let $r_{i}$ be the importance of alliance member $i$ in the alliance, and $\sum_{i=1}^{n} r_{i}=1$. It is mainly derived from the comprehensive evaluation of alliance members' position in the collaborative innovation network and the role of the innovation chain.

Therefore, under the goal of incentive effect, the satisfaction model of alliance members on the benefit distribution of collaborative innovation is as follows: 


$$
\begin{gathered}
\max Z=\prod_{i=1}^{n}\left(\frac{\theta_{i}}{\theta_{i}^{+}}-\frac{\theta_{i}^{-}}{\theta_{i}^{+}}\right)^{r_{i}} \\
\text { s.t. }\left\{\begin{array}{c}
\theta_{i}^{-} \leq \theta_{i} \leq \theta_{i}^{+} \\
\sum_{i=1}^{n} \theta_{i}=1
\end{array}\right.
\end{gathered}
$$

By solving the objective function $\max Z$, find out the optimal benefit distribution coefficient of the cooperative innovation members of the alliance. Among them, $\frac{\theta_{i}}{\theta_{i}^{+}}$is the final satisfaction of the satisfied alliance members, and $\frac{\theta_{i}}{\theta_{i}^{+}}-\frac{\theta_{i}^{-}}{\theta_{i}^{+}}$is the difference between the finality of member $i$ and the minimum expected satisfaction, representing the perceived value brought on the basis of the minimum expected satisfaction. Therefore, the greater the difference, the greater the marginal effect of incentives for members to participate in collaborative innovation. It can be concluded that:

$$
\theta_{i}^{*}=\theta_{i}^{-}+\left(1-\sum_{i=1}^{n} \theta_{i}^{-}\right) \frac{r_{i} \theta_{i}^{+}}{\theta_{i}^{+} \sum_{i=1}^{n} r_{i}}=\theta_{i}^{-}+\left(1-\sum_{i=1}^{n} \theta_{i}^{-}\right) \frac{r_{i}}{\sum_{i=1}^{n} r_{i}},(i=1,2, \cdots, n)
$$

After the optimization process of the benefit distribution coefficient $\theta_{i}$, it can fully realize the incentive effect on the effort of alliance members in the initial stage of benefit distribution, and meet the needs of members for return on investment.

\section{Conclusion}

In this paper, the demand of alliance members for collaborative innovation benefit distribution is divided into risk compensation and investment return, and the corresponding benefit distribution model is designed, which will fully realize the incentive effect of benefit distribution model in each stage on members participating in collaborative innovation. In the stage of risk compensation, by dividing the ownership of the staged achievements, the innovation achievements of members participating in collaborative innovation can be fully guaranteed, and the confidence and motivation of members participating in collaborative innovation can be stimulated; After alliance collaborative innovation achieves innovation performance, members' demand for benefit distribution first focuses on the profit pursuit of all input resources, which will have an important incentive effect on alliance members' efforts to participate in collaborative innovation, and encourage members to invest more resources in collaborative innovation process, and lay a foundation for the establishment of trust relationship among members. Therefore, through the incentive process of members' participation in collaborative innovation efforts and interaction intensity, the smooth progress of collaborative innovation cooperation mode can be fully guaranteed, which plays an important guiding role for members.

To sum up, the benefit distribution mechanism designed in this paper can motivate members under different needs in different stages of collaborative innovation, and can always keep the incentive function for members, thus ensuring the efficient, stable and continuous operation of alliance collaborative innovation.

This work was partially supported by the National Natural Science Foundation of China (No. 71704036), the Philosophy and Social Science Planning Project of Heilongjiang Province (No. 20GLB119; 20JYB033), and the Outstanding Young Talent Project of Talents Plan of Harbin University of Science and Technology (No.2019-KYYWF-0216). 


\section{References}

1. X.Y. Wang and Y.L. Wang. Scientific research management, 3, 32, 29-35, (2011).

2. Q. Zhou, W.J. Zhang, X.Y. Yu. Research and Development Management, 6, 27, (2015).

3. OKAMURO H. Research Policy, 12, 36 , 1529-1544, (2007).

4. X. Wang, W.D. Meng, G.D. Wu. Sys Eng, 7, 32, 63-68, (2014) .

5. G. Li. J Inf, 2, 29, 115-117, (2010).

6. B. Huang, W.D. Meng, Y.Y. Li, etc. J Ind Eng and Eng Man, 4, 24, 58-65, (2010).

7. W AMALDOSS, R J MEYER, et al. Market Sci, 2, 19, (2000).

8. Encarnación Algaba,Vito Fragnelli,. Eur J Oper Res,(2018).

9. Z.J. Wang, H. Hu, Q.C. Zeng, X.B. Li. J Transp Econ Policy, 3, 50,(2016). 